\title{
Relationship between mismatch repair immunophenotype and long-term survival in patients with resected periampullary adenocarcinoma
}

Margareta Heby ${ }^{*}$ (D, Sebastian Lundgren, Björn Nodin, Jacob Elebro, Jakob Eberhard and Karin Jirström

\begin{abstract}
Background: Periampullary adenocarcinomas, including pancreatic cancer, are a heterogeneous group of tumors with poor prognosis, where classification into intestinal type (I-type) or pancreatobiliary type (PB-type) is a relevant prognostic factor. The clinical significance of deficient mismatch repair (dMMR) in periampullary adenocarcinoma is comparatively unexplored. Herein, we examined the associations of MMR immunophenotype with long-term survival in patients with resected periampullary adenocarcinoma, with particular reference to morphology and adjuvant treatment response.

Methods: MMR protein expression was assessed by immunohistochemistry on tissue microarrays with primary tumors from a retrospective cohort of 175 patients with periampullary adenocarcinoma treated with pancreaticoduodenectomy during 2001-2011 in Malmö and Lund University Hospitals, Sweden. Cox proportional hazards models were applied to calculate hazard ratios (HR) and 95\% confidence intervals (Cl).

Results: After a mean follow-up of 46.5 (1.9-185.1) months, 35 patients (20.3\%) were alive, 24 with I-type and 11 with PB-type tumors. MMR protein expression could be evaluated in 172 cases, in which dMMR was denoted in 20 (11.6\%) cases, 13/63 (20.6\%) in l-type and 7/109 (6.4\%) in PB-type tumors. dMMR was associated with a significantly prolonged overall survival in the entire cohort $(H R=0.28,95 \% \mathrm{Cl} 0.13-0.57)$, and in l-type tumors $(\mathrm{HR}=0.20,95 \% \mathrm{Cl}$ 0.06-0.68), however not independent of conventional prognostic factors. In PB-type tumors, dMMR was not prognostic, but there was a significant negative interaction between $\mathrm{dMMR}$ and adjuvant treatment $\left(\mathrm{p}_{\text {interaction }}=0.015\right)$.

Conclusions: $\mathrm{dMMR}$ is more frequent in l-type compared to PB-type periampullary adenocarcinoma, and is a prognostic factor for long-term survival only in the former. The finding of the small number of PB-type tumors with dMMR potentially lacking benefit from adjuvant chemotherapy is however noteworthy and merits further validation.
\end{abstract}

Keywords: MMR, Periampullary adenocarcinoma, TMA, Immunohistochemistry, Adjuvant therapy

\footnotetext{
*Correspondence: margareta.heby@med.lu.se

Department of Clinical Sciences Lund, Division of Oncology

and Pathology, Lund University, Skåne University Hospital, 22185 Lund,

Sweden
} 


\section{Background}

The periampullary region describes the anatomical location around the ampulla of Vater. Adenocarcinoma of this region includes tumors originating in the distal bile duct, pancreas, ampulla of Vater and the periampullary duodenum. These tumors are a heterogeneous group of neoplasms, with pancreatic cancer being the most common type. The periampullary adenocarcinomas are divided into two different morphological types, i.e. pancreatobiliary type (PB-type), and intestinal type (I-type). PB-type tumors, which include pancreatic cancer, distal bile duct cancer, and some of the ampullary carcinomas, have a worse prognosis and are associated with significantly shorter survival rates compared to I-type tumors $[1,2]$, which include duodenal carcinoma and some of the ampullary carcinomas. Hence, tumor morphology provides important prognostic information. The overall 5 -year survival is $7 \%$ for all stages combined and the median survival is approximately 6 months [3]. In R0-resected pancreatic cancer the 5-year survival is $20 \%$ with a median survival of 24 months after resection, whereas for ampullary adenocarcinoma the prognosis is somewhat better with a median survival of 36-44 months after resection [4-6]. Tumors in this region are mainly diagnosed at a late stage, with only $15-20 \%$ being resectable at presentation [7], resectability often being limited by early local invasion of the surrounding anatomical structures such as arterial vessels or distant metastasis. The only cure is surgery with R0 resection, and for borderline resectable tumors neoadjuvant chemotherapy should be offered since approximately one-third of these tumors can be converted to resectability [8]. Randomized trials in the 1990s showed that adjuvant chemotherapy for pancreatic cancer prolongs life, as compared to observation $[9,10]$. Several studies have confirmed these results and adjuvant treatment has now become standard of care. In the palliative setting, comprising almost $80 \%$ of the cases, treatment consists of different chemotherapy combinations and/or radiotherapy, however the effect is often minimal and short-lived. Therefore, there is a great need for additional molecular-based biomarkers, to better define clinically relevant subgroups of these tumors, so as to enable improved personalized treatment strategies. Immune-modulating therapy, e.g. targeting the programmed death receptor 1 (PD-1) pathway, is a treatment option that has shown promising results in various types of tumors, but the efficacy in periampullary cancer remains unclear and checkpoint inhibition in pancreatic cancer has been disappointing thus far $[11,12]$. Mismatch repair (MMR) immunophenotype, is a putative biomarker of response to such therapies. Epigenetic or mutational inactivation of certain MMR genes, including MutL homolog 1 (MLH1), post-meiotic segregation 2 (PMS2), MutS protein homolog 2 (MSH2) and MutS protein homolog 6 (MSH6), typically results in microsatellite instability (MSI) which means a failure to repair errors that occur during replication of repetitive DNA sequences [13]. Consequently, these tumors contain thousands of mutations that may produce neoantigens that can be recognized and targeted by $\mathrm{T}$ cells, changes that have been linked to increased sensitivity to checkpoint inhibitors, e g programmed death receptor 1 (PD-1) blockade [14]. MMR deficient tumors have been demonstrated to respond to treatment with the anti-PD-1 immune checkpoint inhibitor pembrolizumab [15]. In colorectal cancer (CRC), high levels of MSI have been found to predict a better overall prognosis and an increased benefit from immune based therapies [16], however there are reports suggesting that in stage IV disease, the prognosis of CRC with high MSI is poorer than for microsatellite-stable cases [17]. The prevalence of dMMR and high MSI in pancreatic cancer has in some studies been reported to range between 13 and $22 \%$ in primarily surgically resected patients $[18,19]$, but still remains poorly characterized. Some recently published studies conclude that MSI positivity in resectable pancreatic cancer may be a favorable prognostic factor $[18$, 20], and one study on ampulla of Vater adenocarcinoma demonstrated that patients with MSI high tumors had a significantly longer overall survival [21]. In conclusion, the role of MMR immunophenotype as a potential prognostic and predictive biomarker for response to adjuvant and immunotherapy in periampullary adenocarcinoma remains unclear, hence further investigation is warranted. The aim of this study was therefore to examine the frequency of MMR deficiency in a retrospective cohort of periampullary adenocarcinoma, with particular reference to tumor morphology and relationship with long-term survival and adjuvant treatment response.

\section{Methods \\ Patients}

The study cohort consists of a previously described retrospective consecutive series of 175 patients with primary periampullary adenocarcinomas [22-26]. All patients were subjected to pancreaticoduodenectomy at the University hospitals of Lund and Malmö, Sweden, from January 12001 until December 31 2011. In the full cohort of 175 cases the anatomical origin was 14 duodenal, 70 ampullary, 45 distal bile duct and 46 pancreatic, in all 110 PB-type and 65 I-type adenocarcinomas. Data on survival were gathered from the Swedish National Civil Register. Follow-up started at the date of surgery and ended at death, or at March 31 2017, whichever came first. Clinical data regarding adjuvant treatment, recurrence and clinicopathological data were obtained 
retrospectively from medical records and the last update reaches until March 31 2017. The patients were identified through broad searches in the pathology database, and all haematoxylin and eosin stained slides were re-evaluated by one pathologist (JEL), blinded to the original report and outcome, with the decision on tumor origin and morphological type being based on several criteria, as previously described [22].

The study was approved by the Ethics Committee of Lund University (ref no. 445/07).

\section{Tissue microarray construction}

Tissue microarrays (TMAs) were constructed using a semi-automated arraying device (TMArrayer, Pathology Devices, Westminister, MD, USA). A standard set of three tissue cores $(1 \mathrm{~mm})$ were obtained from each of the 175 primary tumors and from lymph node metastases from 105 of the cases, whereby one to three lymph node metastases were sampled in each case. In addition, adjacent benign-appearing pancreatic tissue was sampled from 50 cases using two $(1 \mathrm{~mm})$ tissue cores.

\section{Immunohistochemistry and staining evaluation}

For immunohistochemical analysis of MMR proteins, $4 \mu \mathrm{m}$ TMA sections were automatically pretreated in the PT-link system (Dako, Glostrup, Denmark) with heat induced epitope retrieval (HIER) in TRS pH 9 (Dako, catalogue $\mathrm{nr} \mathrm{K8004),} 20 \mathrm{~min}$ in $97^{\circ} \mathrm{C}$, and then stained in an automated immunostainer (Autostainer Link 48, Dako) using the Dako EnVision ${ }^{\mathrm{TM}} \mathrm{FLEX}+$ Detection System, Peroxidase/DAB, Rabbit/Mouse (catalogue nr K8002), with the following ready- to -use monoclonal antibodies: MLH1 (clone ES05, part nr IR07961-2, Dako), PMS2 (clone EP51, part nr IR8761-2, Dako), MSH2 (clone FE11, part nr IR08561-2, Dako) and MSH6 (clone EP49, part nr IR08661-2, Dako). After IHC staining, slides were rinsed 5 min in tap water, dehydrated and mounted with Tissue Tek Prisma (Sakura Finetek, Alphen aan den Rijn, Netherlands).

Staining of MMR was evaluated by three independent observers (MH, SL and $\mathrm{KJ})$, who were blinded to clinical and outcome data. Immunohistochemical stainings were denoted as negative when all tumor cells showed loss of nuclear staining. Surrounding stromal cells and tumor infiltrating lymphocytes served as internal controls for each TMA core. Cases lacking positive internal controls were excluded. Deficient MMR (dMMR) was defined as negative staining for MLH1, PMS2, MSH2 or MSH6, and proficient MMR (pMMR) was defined as positive staining for all four MMR proteins. Immunohistochemical analysis of $\mathrm{CD}^{+}$lymphocytes, $\mathrm{CD} 56^{+}$natural killer $(\mathrm{NK}) / \mathrm{NKT}$ cells and $\mathrm{CD}^{+} 8^{+}$and $\mathrm{CD} 163^{+}$macrophages had been performed previously [27, 28]. For immunohistochemical analysis of FoxP3 and $\mathrm{CD}^{+}{ }^{+} \mathrm{T}$ cells, $4 \mu \mathrm{m}$ TMA-sections were automatically pre-treated using the PT Link system and then stained in an Autostainer Plus (Dako; Glostrup, Denmark) with the anti-FoxP3 antibody (clone 236A/E7, mouse, dilution 1:200, Abcam, Cambridge, UK), and the anti-CD8 antibody (clone C8/144B, mouse; dilution, 1:50; product M7103; Dako). The total number (intratumoral, tumor-adjacent and stromal) $\mathrm{CD}^{+}$immune cells in each core was calculated by automated analysis using the co-localization algorithm within the Halo image analysis software (Indica Labs, Corrales, $\mathrm{NM}, \mathrm{USA}$ ). The number of FoxP3 ${ }^{+}$cells (intratumoral, tumor-adjacent and stromal) was calculated manually. A median value of the cores was calculated and used in the analyses.

\section{Statistical analysis}

Chi square test was applied to analyze the relationship between MMR immunophenotype and categorical clinicopathological parameters, whereas Mann-Whitney U test was used in continuous variables such as age, tumor size and immune cells. Three patients were excluded from the survival analyses; two with I-type adenocarcinomas who died within 1 month from surgery due to complications and one with PB-type adenocarcinoma who emigrated 5 months after surgery. Kaplan-Meier analysis and log rank test were applied to estimate differences in long-term overall survival (OS), in strata according to dMMR/pMMR. Hazard ratios (HR) for death and recurrence were calculated by Cox regression proportional hazard's modeling in unadjusted analysis and in a multivariable model. Only factors with a $p$ value $<0.05$ were included in the multivariable analysis. To estimate the interaction effect between adjuvant treatment and MMR immunophenotype, the following interaction variable was constructed; any adjuvant treatment $( \pm) \times$ MSI $( \pm)$. The proportional hazard $(\mathrm{PH})$ assumption was tested using Cox regression with a time-dependent covariate analysis, whereby the $\mathrm{PH}$ assumption was considered to be satisfied when the factor $\times$ time interaction was nonsignificant. The $\mathrm{PH}$ assumption was also evaluated graphically using log-minus-log plots. All tests were two sided. p-values $<0.05$ were considered significant. All statistical analyses were performed using IBM SPSS Statistics version 22.0 (SPSS Inc., Chicago, IL, USA).

\section{Results}

Three patients were excluded from the survival analyses; two with I-type adenocarcinomas who died within 1 month from surgery due to complications and one with PB-type adenocarcinoma who emigrated 5 months 
after surgery. Mean and median follow-up time was 46.5 and 29.7 months (Q1 16.6 and Q3 65.6 months) respectively (range 1.9-185.1 months), and in March 2017, 35 patients $(20.3 \%)$ were alive, 24 with I-type and 11 with PB-type tumors. Recurrent disease was denoted in 124 patients (72.1\%), 31 with I-type and 93 with PB-type tumors. Thus, $50.8 \%$ of patients with I-type tumors did not have recurrence at follow-up in March 2017. Two of the 24 patients alive with I-type tumor morphology had recurrent disease and were of pMMR immunophenotype. Ten patients with I-type and 5 patients with PBtype tumors died without signs of recurrence and thus from other causes. Adjuvant chemotherapy was given in 77 (44.8\%) cases, 18/63 (28.6\%) I-type and 59/109 (54.1\%) PB-type tumors. Of patients with I-type tumors, $50 \%$ received 5-fluorouracil (5-FU)-based chemotherapy, and $50 \%$ gemcitabine-based chemotherapy, single or in combination. Corresponding numbers in patients with PBtype tumors were less than 20\% 5-FU-based and the rest gemcitabine-based treatment.

\section{MMR immunophenotype}

Sample immunohistochemical images of MMR protein expression are shown in Fig. 1. MMR protein expression could be evaluated in 172 cases, in which dMMR was denoted in $20(11.6 \%)$ cases, 13/63 (20.6\%) in I-type and $7 / 109$ (6.4\%) in PB-type tumors. The distribution of loss of different MMR proteins in relation to morphological type is shown in Table 1. Loss of MSH6 was all over the most common, and seen in $71.4 \%$ of PB-type tumors. In I-type tumors the distribution of different combinations was more even. The distribution of MMR immunophenotype according to anatomical subsite is presented in Fig. 2. In tumors with duodenal origin, $43 \%$ were denoted as having dMMR. There was no discordance in MMR immunophenotype between primary tumors and lymph node metastases. In the two cases that had received neoadjuvant chemotherapy, both tumors were pMMR and thus included in the analyses, since neoadjuvant
Table 1 The distribution of loss of different MMR proteins in relation to morphological type

\begin{tabular}{|c|c|c|c|c|}
\hline n (\%) & MLH1 & PMS2 & MSH2 & MSH6 \\
\hline \multicolumn{5}{|l|}{ All } \\
\hline $1(5.0)$ & Intact & Loss & Intact & Uninterpretable \\
\hline $1(5.0)$ & LosS & LosS & Intact & Loss \\
\hline $3(15.0)$ & LosS & LosS & Intact & Intact \\
\hline $4(20.0)$ & Intact & Loss & Intact & Intact \\
\hline $3(15.0)$ & Intact & Intact & Loss & Loss \\
\hline $8(40.0)$ & Intact & Intact & Intact & Loss \\
\hline \multicolumn{5}{|l|}{ I-type } \\
\hline $1(7.7)$ & Loss & Loss & Intact & Loss \\
\hline $3(23.1)$ & Loss & Loss & Intact & Intact \\
\hline $4(30.1)$ & Intact & Loss & Intact & Intact \\
\hline $2(15.4)$ & Intact & Intact & Loss & Loss \\
\hline $3(23.1)$ & Intact & Intact & Intact & Loss \\
\hline \multicolumn{5}{|l|}{ PB-type } \\
\hline $1(14.3)$ & Intact & Loss & Intact & Uninterpretable \\
\hline $1(14.3)$ & Intact & Intact & Loss & Loss \\
\hline $5(71.4)$ & Intact & Intact & Intact & Loss \\
\hline
\end{tabular}

${ }^{a}$ Lack of positive internal control staining in lymphocytes or stromal cells

treatment had not affected the expression of any MMR protein.

\section{Associations of MMR immunophenotype with clinicopathological factors and tumor-infiltrating immune cells}

The associations of MMR immunophenotype with clinicopathological factors and tumor-infiltrating immune cells in the entire cohort, I-type and PB-type tumors, respectively, are shown in Table 2. In the entire cohort, dMMR was significantly associated with I-type morphology $(\mathrm{p}=0.003)$, N0-stage $(\mathrm{p}=0.002)$, perineural growth $(\mathrm{p}=0.003)$, absence of tumor growth in lymphatic vessels $(\mathrm{p}=<0.001)$ and in peripancreatic fat $(\mathrm{p}=0.002)$, infiltration of $\mathrm{CD}^{+} \mathrm{T}$ cells $(\mathrm{p}=0.035)$, infiltration of $\mathrm{CD} 56^{+}$ cells $(\mathrm{p}=0.029)$ and with no adjuvant chemotherapy

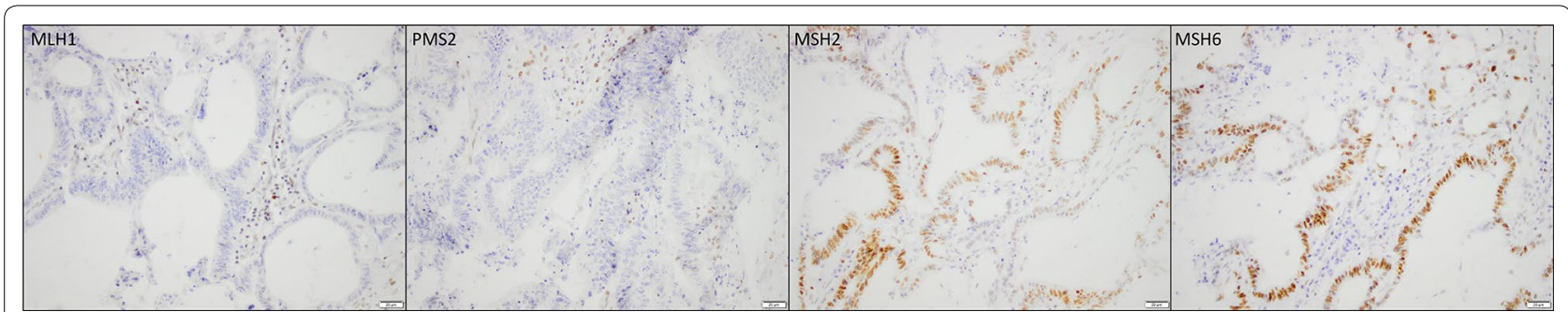

Fig. 1 Sample immunohistochemical images of MMR protein expression in an MMR deficient duodenal cancer (MHL1 and PMS2 negative, MSH2 and MSH6 positive) 


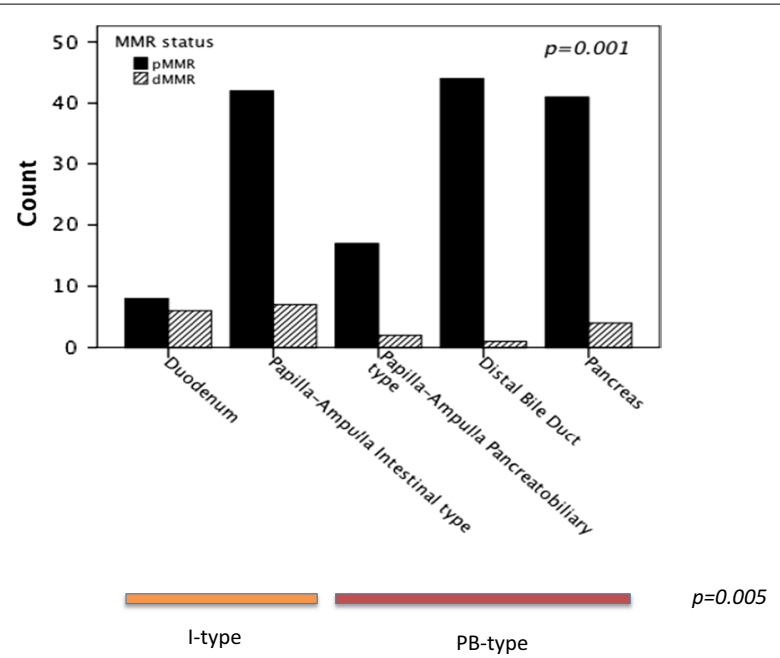

Fig. 2 The distribution of MMR immunophenotype according to anatomical subsite

$(\mathrm{p}=0.040)$. When divided into morphological subtype, dMMR was significantly associated with tumor origin in the duodenum $(\mathrm{p}=0.005)$, larger tumor size $(\mathrm{p}=0.006)$, absence of tumor growth in lymphatic vessels $(p=0.002)$, and infiltration of $\mathrm{CD}^{+} \mathrm{T}$ cells $(\mathrm{p}=0.012)$ in I-type tumors. In PB-type tumors there was a significant association between $\mathrm{dMMR}$ and N0-stage $(\mathrm{p}=0.002)$, absence of tumor growth in lymphatic vessels $(\mathrm{p}=0.018)$ and in peripancreatic fat $(\mathrm{p}=0.021)$, and infiltration of $\mathrm{CD} 56^{+}$ cells $(\mathrm{p}=0.046)$. The density of $\mathrm{CD} 8^{+} \mathrm{T}$ cells and CD56 ${ }^{+}$ NK/NKT cells was significantly higher in dMMR tumors, but there were no significant associations between $\mathrm{dMMR}$ and the other investigated immune cell subsets. Sample immunohistochemical images of CD8+and FoxP3 lymphocytes in the same tumor as in Fig. 1 are shown in Fig. 3.

\section{Prognostic and potential predictive value of MMR immunophenotype}

As demonstrated in Fig. 4, Kaplan-Meier analysis revealed a significant association between dMMR and prolonged overall survival in the entire cohort $(\mathrm{p}=<0.001)$ and in I-type tumors $(\mathrm{p}=0.004)$, but not in PB-type tumors. Cox proportional hazards analyses of OS according to MMR immunophenotype are shown in Table 3. The time-dependent covariate was non-significant for MMR immunophenotype, and therefore, the factor $\times$ time interaction term was dropped from the model. The proportional hazard assumption was also considered to be satisfied with graphical evaluation using log-minuslog plots (data not shown). The associations between MMR immunophenotype and OS were confirmed in univariable Cox regression analysis in the entire cohort
$(\mathrm{HR}=0.28,95 \% \mathrm{CI} 0.13-0.57)$ and in I-type tumors $(\mathrm{HR}=0.20,95 \% \mathrm{CI} 0.06-0.68)$. However, none of these associations were significant when adjusted for conventional prognostic factors. Similar findings were seen for RFS according to MMR immunophenotype, with significant values in the entire cohort and in I-type tumors, but not in PB-type tumors, and not when adjusted for conventional prognostic factors (Additional file 1). KaplanMeier analysis of overall survival in strata according to MMR immunophenotype and adjuvant treatment is presented in Fig. 5. In the entire cohort (Fig. 5a) and in PBtype tumors (Fig. 5b), patients with dMMR tumors who had not received adjuvant chemotherapy had the best prognosis, and in I-type tumors, patients with dMMR tumors had the best prognosis irrespective of adjuvant chemotherapy (Fig. 5c). However, and notably, patients with $\mathrm{dMMR}$ PB-type tumors who had received adjuvant treatment had the shortest OS (Fig. 5b), and there was a significant negative interaction between $\mathrm{dMMR}$ and adjuvant treatment $\left(\mathrm{p}_{\text {interaction }}=0.015\right)$.

\section{Discussion}

Despite improvements in the treatment of other solid tumors, e.g. with immune-modulating therapies, overall survival for patients with periampullary adenocarcinoma, continues to be poor. By year 2030, pancreatic adenocarcinoma is predicted to become the second leading cause of cancer death after lung/bronchus cancer [29, 30]. Apart from the prognostic information provided by standard histopathological parameters and CA19-9, no prognostic or predictive biomarkers have yet been introduced into clinical practice. Thus, there is a great need to identify additional biomarkers for improved treatment stratification of patients with periampullary adenocarcinoma, including pancreatic cancer. In this study, we evaluated the prognostic and predictive impact of MMR immunophenotype in periampullary adenocarcinoma with regard to morphological type and adjuvant therapy. In the herein investigated cohort, dMMR was denoted in $11.6 \%$ of the cases, which is in line with findings in previous studies $[18,19]$. MMR proteins functionally interact in heterodimers, where MLH1 and MSH2 are obligatory proteins in their respective heterodimer and their mutational or epigenetic inactivation leads to destabilization of the corresponding binding partners and results in complete loss of MMR activity [31]. However, other immunohistochemical patterns have been described in pancreatic cancer with loss of MSH2 but not MSH6 [18] and in Lynch syndrome, where the multitude of disease-predisposing mutations may have variable effects on epitope expression, from complete loss to weak or retained expression for one or both heterodimerizing proteins [32, 33]. Since limitations to 
Table 2 The association between MMR immunophenotype with clinicopathological factors in I-type tumors, PB-type tumors, and the entire cohort respectively

\begin{tabular}{|c|c|c|c|c|c|c|c|c|c|}
\hline & \multicolumn{3}{|l|}{ Intestinal type } & \multicolumn{3}{|c|}{ Pancreatobiliary type } & \multicolumn{3}{|l|}{ All } \\
\hline & $\begin{array}{l}\text { pMMR } \\
(n=50)\end{array}$ & $\begin{array}{l}\text { dMMR } \\
(n=13)\end{array}$ & $p$ & $\begin{array}{l}\text { pMMR } \\
(n=102)\end{array}$ & $\begin{array}{l}\mathrm{dMMR} \\
(\mathrm{n}=7)\end{array}$ & $p$ & $\begin{array}{l}\text { pMMR } \\
(n=152)\end{array}$ & $\begin{array}{l}\text { dMMR } \\
(n=20)\end{array}$ & $p$ \\
\hline \multicolumn{10}{|l|}{ Age } \\
\hline (Median, range) & $66.5(38.0-79.0)$ & $67.0(48.0-83.0)$ & 0.905 & $67.0(44.0-81.0)$ & $62.0(58.0-76.0)$ & 0.669 & $67.0(38.0-81.0)$ & $65.5(48.0-83.0)$ & 0.635 \\
\hline \multicolumn{10}{|l|}{ Sex } \\
\hline Women & $26(52.0)$ & $8(61.5)$ & 0.539 & $46(45.1)$ & $4(57.1)$ & 0.538 & $72(47.4)$ & $12(60.0)$ & 0.289 \\
\hline Men & $24(48.0)$ & $5(38.5)$ & & $56(54.9)$ & $3(42.9)$ & & $80(52.6)$ & $8(40.0)$ & \\
\hline \multicolumn{10}{|l|}{ Tumor origin } \\
\hline Duodenum & $8(16.0)$ & $6(46.2)$ & 0.005 & & & & $8(5.3)$ & $6(28.6)$ & 0.003 \\
\hline $\begin{array}{l}\text { Ampulla intestinal } \\
\text { type }\end{array}$ & $42(84.0)$ & $7(53.8)$ & & & & & $42(27.6)$ & $7(35.0)$ & \\
\hline $\begin{array}{l}\text { Ampulla pancreatobil- } \\
\text { iary type }\end{array}$ & & & & $17(16.7)$ & $2(28.6)$ & 0.860 & $17(11.2)$ & $2(10.0)$ & \\
\hline Distal bile duct & & & & $44(43.1)$ & $1(14.3)$ & & $44(28.9)$ & $1(5.0)$ & \\
\hline Pancreas & & & & $41(40.2)$ & $4(57.1)$ & & $41(27.0)$ & $4(20.0)$ & \\
\hline \multicolumn{10}{|l|}{ Tumor size mm } \\
\hline (Median, range) & $25.0(5.0-60.0)$ & $40.0(13.0-90.0)$ & 0.006 & $30.0(9.0-70.0)$ & $30.0(5.0-35.0)$ & 0.283 & $30.0(5.0-70.0)$ & $33.0(5.0-90.0)$ & 0.107 \\
\hline \multicolumn{10}{|l|}{ Differentiation grade } \\
\hline Well-moderate & $24(48.0)$ & $7(53.8)$ & 0.707 & $37(36.3)$ & $4(57.1)$ & 0.272 & $61(40.1)$ & $11(55.0)$ & 0.206 \\
\hline Poor & $26(52.0)$ & $6(46.2)$ & & $65(63.7)$ & $3(42.9)$ & & $91(59.9)$ & $9(45.0)$ & \\
\hline \multicolumn{10}{|l|}{ T-stage } \\
\hline $\mathrm{T} 1$ & $4(8.0)$ & 0 & 0.736 & $2(2.0)$ & 0 & 0.966 & $6(3.9)$ & 0 & 0.420 \\
\hline $\mathrm{T} 2$ & $9(18.0)$ & $2(15.4)$ & & $11(10.8)$ & $1(14.3)$ & & $20(13.2)$ & $3(15.0)$ & \\
\hline T3 & $19(38.0)$ & $6(46.2)$ & & 73 (71.6) & $5(71.4)$ & & $92(60.5)$ & $11(55.0)$ & \\
\hline $\mathrm{T} 4$ & $18(36.0)$ & $5(38.5)$ & & $16(15.7)$ & $1(14.3)$ & & $34(22.4)$ & $6(30.0)$ & \\
\hline \multicolumn{10}{|l|}{$\mathrm{N}$-stage } \\
\hline No & $24(48.0)$ & $9(69.2)$ & 0.348 & $26(25.5)$ & $6(85.7)$ & 0.002 & $50(32.9)$ & $15(75.0)$ & 0.002 \\
\hline N1 & $17(34.0)$ & $2(15.4)$ & & $44(43.1)$ & $1(14.3)$ & & $61(40.1)$ & $3(15.0)$ & \\
\hline $\mathrm{N} 2$ & $9(18.0)$ & $2(15.4)$ & & $32(31.4)$ & 0 & & $41(27.0)$ & $2(10.0)$ & \\
\hline \multicolumn{10}{|l|}{ Margins } \\
\hline Ro & $13(26.0)$ & $4(30.8)$ & 0.730 & $6(5.9)$ & 0 & 0.511 & $19(12.5)$ & $4(20.0)$ & 0.356 \\
\hline $\mathrm{R} 1-\mathrm{Rx}$ & $37(74.0)$ & $9(69.2)$ & & $96(94.1)$ & $7(100.0)$ & & $133(87.5)$ & $16(80.0)$ & \\
\hline \multicolumn{10}{|l|}{ Perineural growth } \\
\hline No & $33(66.0)$ & $11(84.6)$ & 0.193 & $21(20.6)$ & $3(42.9)$ & 0.171 & $54(35.5)$ & $14(70.0)$ & 0.003 \\
\hline Yes & $17(34.0)$ & $2(15.4)$ & & $81(79.4)$ & $4(57.1)$ & & $98(64.5)$ & $6(30.0)$ & \\
\hline \multicolumn{10}{|c|}{ Invasion of lymphatic vessels } \\
\hline No & $18(36.0)$ & $11(84.6)$ & 0.002 & $29(28.4)$ & $5(71.4)$ & 0.018 & $47(30.9)$ & $16(80.0)$ & 0.000 \\
\hline Yes & $32(64.0)$ & $2(15.4)$ & & $72(71.6)$ & $2(28.6)$ & & $105(69.1)$ & $4(20.0)$ & \\
\hline \multicolumn{10}{|c|}{ Invasion of blood vessels } \\
\hline No & $45(90.0)$ & $13(100.0)$ & 0.235 & $67(65.7)$ & $5(71.4)$ & 0.757 & $112(73.7)$ & $18(90.0)$ & 0.111 \\
\hline Yes & $5(10.0)$ & 0 & & $35(3437)$ & $2(28.6)$ & & $40(26.3)$ & $2(10.0)$ & \\
\hline \multicolumn{10}{|c|}{ Growth in peripancreatic fat } \\
\hline No & $31(62.0)$ & $10(76.9)$ & 0.315 & $20(19.6)$ & $4(57.1)$ & 0.021 & $51(33.6)$ & $14(70.0)$ & 0.002 \\
\hline Yes & $19(38.0)$ & $3(23.1)$ & & $82(80.4)$ & $3(42.9)$ & & $101(66.4)$ & $6(30.0)$ & \\
\hline \multicolumn{10}{|l|}{ Adjuvant chemotherapy } \\
\hline None & $35(70.0)$ & 10 (76.9) & 0.094 & $46(45.1)$ & $4(57.1)$ & 0.177 & $81(53.3)$ & $14(70.0)$ & 0.040 \\
\hline 5FU-analogue & $5(10.0)$ & 0 & & $8(7.8)$ & 0 & & $13(8.6)$ & $0(0.0)$ & \\
\hline Gemcitabine & $7(14.0)$ & 0 & & $43(42.2)$ & $2(28.6)$ & & $50(32.9)$ & $2(10.0)$ & \\
\hline
\end{tabular}


Table 2 (continued)

\begin{tabular}{|c|c|c|c|c|c|c|c|c|c|}
\hline & \multicolumn{3}{|c|}{ Intestinal type } & \multicolumn{3}{|c|}{ Pancreatobiliary type } & \multicolumn{3}{|l|}{ All } \\
\hline & $\begin{array}{l}\text { pMMR } \\
(n=50)\end{array}$ & $\begin{array}{l}\text { dMMR } \\
(n=13)\end{array}$ & $p$ & $\begin{array}{l}\text { pMMR } \\
(n=102)\end{array}$ & $\begin{array}{l}d M M R \\
(n=7)\end{array}$ & $p$ & $\begin{array}{l}\text { pMMR } \\
(n=152)\end{array}$ & $\begin{array}{l}\text { dMMR } \\
(n=20)\end{array}$ & $p$ \\
\hline $\begin{array}{l}\text { Gemcit- } \\
\text { abine + capecit- } \\
\text { abine }\end{array}$ & 0 & $1(7.7)$ & & $3(2.9)$ & 0 & & $3(2.0)$ & $1(5.0)$ & \\
\hline $\begin{array}{l}\text { Oxaliplatin + 5-FU } \\
\text { analogue }\end{array}$ & $2(4.0)$ & $2(15.4)$ & & $1(1.0)$ & 0 & & $3(2.0)$ & $2(10.0)$ & \\
\hline $\begin{array}{l}\text { Gemcitabine + oxali- } \\
\text { platin }\end{array}$ & $1(2.0)$ & 0 & & $1(1.0)$ & $1(12.5)$ & & $2(1.3)$ & $1(5.0)$ & \\
\hline \multicolumn{10}{|l|}{ Immune cells } \\
\hline $\mathrm{CD}^{+}$ & $206(6-795)$ & $246(59-559)$ & 0.156 & $129(22-546)$ & $44(26-695)$ & 0.092 & $148.5(6-795)$ & $227(26-695)$ & 0.471 \\
\hline Missing & 0 & 0 & & 0 & 0 & & 0 & 0 & \\
\hline $\mathrm{CD}^{+}$ & $55(2-180)$ & $114(2-180)$ & 0.012 & $45(4-175)$ & $37(1-200)$ & 0.844 & $47.5(2-180)$ & $101.5(1-200)$ & 0.035 \\
\hline Missing & 1 & 0 & & 3 & 0 & & 4 & 0 & \\
\hline $\mathrm{FOXP3}^{+}$ & $33(0-110)$ & $40(1-103)$ & 0.497 & $26(1-137)$ & $15(0-119)$ & 0.420 & $27.5(0-137)$ & $38(0-119)$ & 0.560 \\
\hline Missing & 0 & 0 & & 2 & 1 & & 2 & 1 & \\
\hline $\mathrm{CD}_{6} 8^{+}$ & $87(29-350)$ & $74(19-182)$ & 0.490 & $98(25-230)$ & $97(36-230)$ & 0.645 & $93(25-350)$ & $82(19-229)$ & 0.455 \\
\hline Missing & 0 & 0 & & 1 & 1 & & 1 & 1 & \\
\hline CD163 ${ }^{+}$ & $130(35-250)$ & $120(44-190)$ & 0.755 & $140(49-275)$ & $162(92-200)$ & 0.241 & $136(35-275)$ & $131(44-200)$ & 0.885 \\
\hline Missing & 1 & 0 & & 4 & 1 & & 5 & 1 & \\
\hline $\mathrm{CD}_{5} 6^{+}$ & $1(0-9)$ & $2(0-23)$ & 0.370 & $1(0-12)$ & $3(0-33)$ & 0.046 & $1(0-12)$ & $2(0-33)$ & 0.029 \\
\hline Missing & 0 & 0 & & 0 & 0 & & 0 & 0 & \\
\hline
\end{tabular}

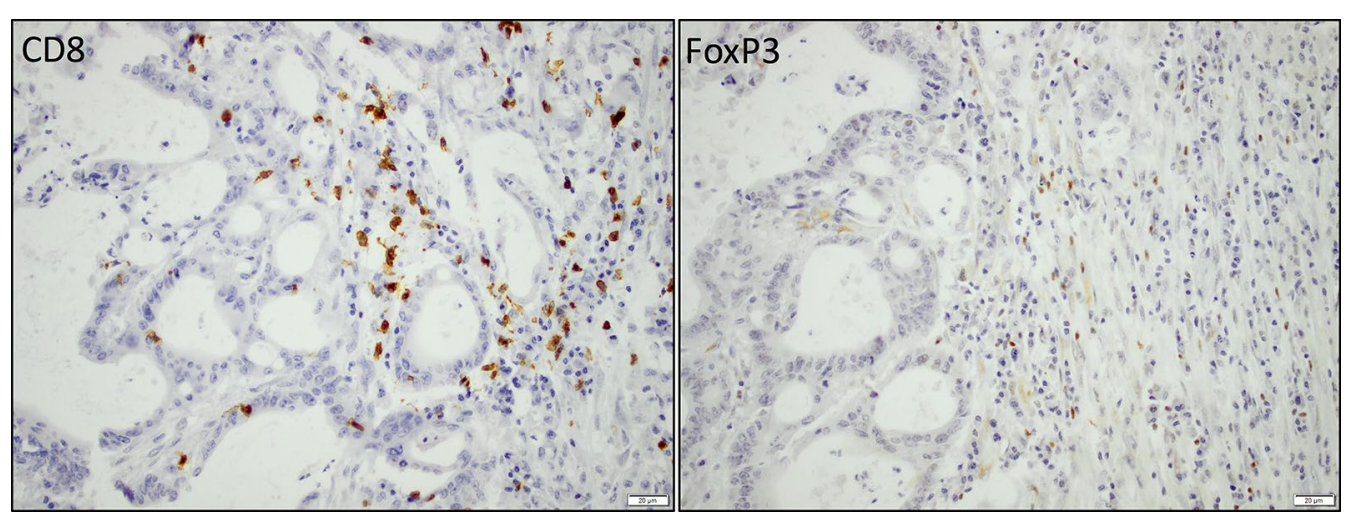

Fig. 3 Sample immunohistochemical images of CD8+ (membranous/cytoplasmic staining) and FoxP3 (nuclear staining) lymphocytes in the same tumor as in Fig. 1

immunohistochemistry exist, such as the antibodies used in the analysis, the staining pattern, the biopsy sample size and the risk of false negative cases, all results should always be interpreted with this in mind. In our study, a false negative sample (interpreted as dMMR) would alter the results profoundly given the small sample size of tumors with dMMR. However, immunohistochemistry is often the first choice for screening to identify patients for genetic testing, and given its fairly low cost and availability, this method is widely used in the clinical setting as opposed to MSI-testing [34]. The majority of cases with PB-type tumors in this study had lost the expression of MSH6, whereas in I-type tumors, the dominating immunophenotype was loss of PMS2 expression. Our results support the previously described association between dMMR and a prolonged survival in patients with pancreatic cancer $[18,20]$ and ampulla of Vater adenocarcinomas [21]. To the best of our knowledge, there are however no studies reporting on whether the clinical impact of MMR immunophenotype in periampullary 


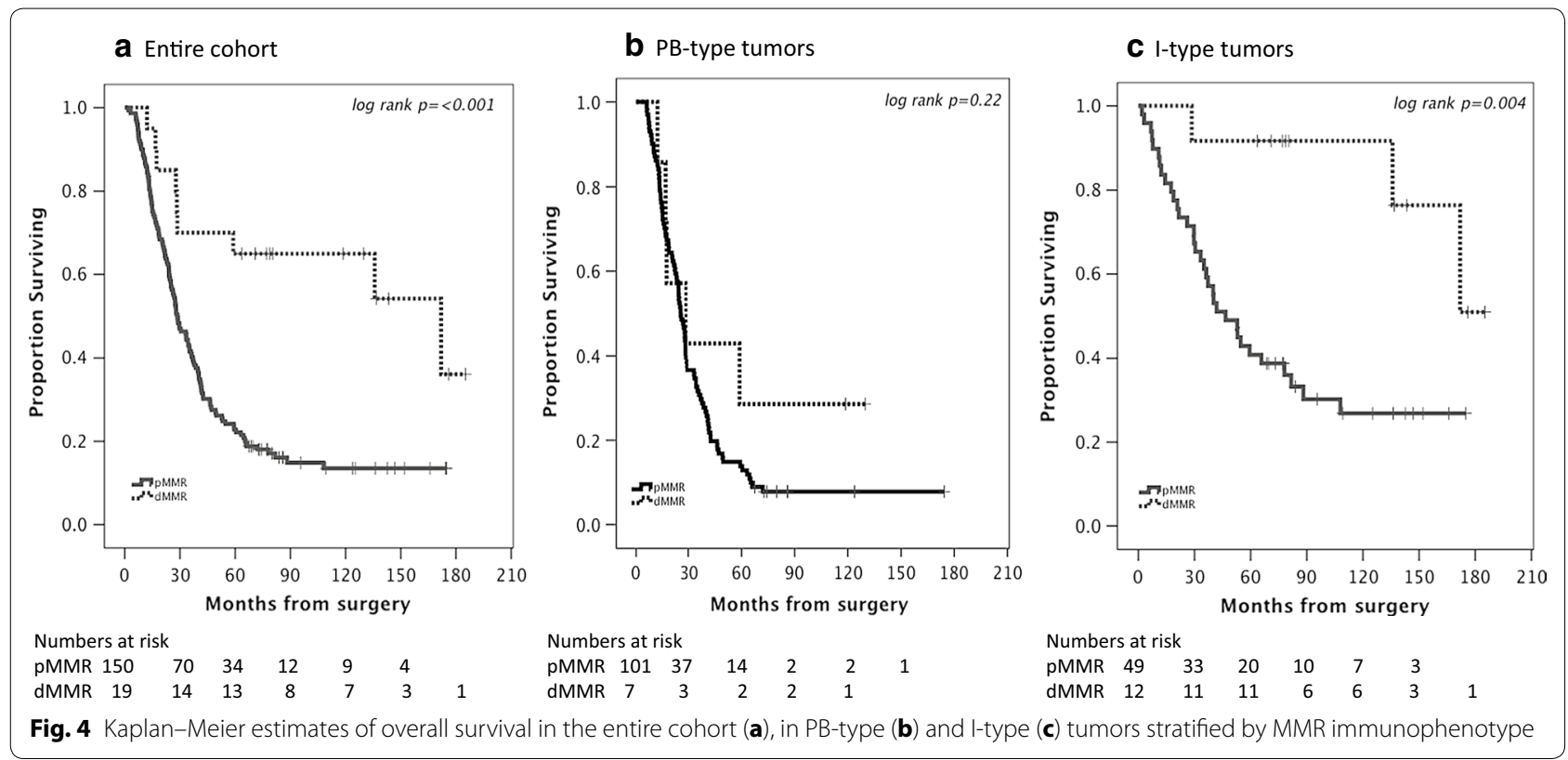

cancer differs by morphological type. I-type tumors bear a resemblance to colorectal cancer, and the results from the present study demonstrate that dMMR is more common in I-type tumors than in PB-type tumors, and confers a prognostic value only in the former, although this did not remain significant in the adjusted model. dMMR was significantly associated with more favorable clinicopathological factors in both I-type and PB-type tumors. Colorectal cancers with dMMR and MSI are known to have a more dense infiltration of intraepithelial activated $\mathrm{CD}^{+}$tumor-infiltrating lymphocytes than microsatellite stable colorectal cancers [34]. Our results also demonstrate an association between $\mathrm{dMMR}$ and $\mathrm{CD} 8^{+}$ tumor-infiltrating lymphocytes in I-type tumors, and an association between dMMR and $\mathrm{CD} 56^{+} \mathrm{NK} / \mathrm{NKT}$ cells in PB-type tumors. A high density of CD $56^{+}$NK/NKT cells has previously been shown to be significantly associated with a prolonged survival in this cohort [28]. Moreover, and notably, there was a negative interaction between high density of $\mathrm{CD} 56^{+} \mathrm{NK} / \mathrm{NKT}$ cells and adjuvant treatment in patients with PB-type tumors, which is similar to the findings related to dMMR status in this study. This observation should however be interpreted with caution, given the small number of cases with dMMR in PB-type tumors, but is still noteworthy and merits validation, since it may be of clinical relevance. This observation is also supported by findings in colorectal cancer, where studies have shown that patients with MSI high tumors have no survival benefit from adjuvant 5-FU-based chemotherapy, and have an even poorer response to 5-FU, compared to patients with pMMR or microsatellite stable tumors $[35,36]$. One theory explaining the resistance to 5-FU in dMMR tumors is the overexpression of tumoral thymidylate synthase (TS), which is the main 5-FU target, and dihydropyrimidine dehydrogenase (DPD), which is the key enzyme of 5-FU catabolism [37]. MMR immunophenotype may indeed confer a predictive value in periampullary adenocarcinoma as Riazy et al. recently demonstrated a prolonged disease-specific survival with gemcitabine or 5-FU treatment in patients with pMMR pancreatic cancer $(\mathrm{n}=224)$, but no statistically significant survival advantage for treated patients with dMMR tumors $(n=41)$ [18]. In the present study it is noteworthy that none of the patients with $\mathrm{dMMR}$ PB-type tumors received 5-FU, which might imply that dMMR also signifies resistance to other types of chemotherapeutic agents. A comparative strength of the herein investigated retrospective patient cohort is that it encompasses a large proportion of patients who did not receive any adjuvant chemotherapy, which is due to the fact that all types of periampullary adenocarcinomas are included and that the cohort stretches from 2001 to 2011, thus spanning a period of time where adjuvant treatment was yet not standard of care. Moreover, the proportion of patients with PB-type tumors in this cohort is comparatively large, with 110 patients, whereof almost 50\% did not receive adjuvant chemotherapy. Notably, there was no significant difference in the distribution of conventional clinicopathological factors between treated and untreated patients, except for a significantly higher age in the latter. Therefore, although treatment predictive effects are best studied in a randomized setting, 
Table 3 Unadjusted and adjusted hazard ratios of the impact of MMR immunophenotype on overall survival in I-type tumors, PB-type tumors, and the entire cohort

\begin{tabular}{|c|c|c|c|c|c|c|c|c|c|}
\hline & \multicolumn{3}{|c|}{ Intestinal type } & \multicolumn{3}{|c|}{ Pancreatobiliary type } & \multicolumn{3}{|l|}{ All } \\
\hline & n (events) & Unadjusted & Adjusted & n (events) & Unadjusted & Adjusted & n (events) & Unadjusted & Adjusted \\
\hline & & $\mathrm{HR}(95 \% \mathrm{Cl})$ & $\mathrm{HR}(95 \% \mathrm{Cl})$ & & $\mathrm{HR}(95 \% \mathrm{Cl})$ & $\mathrm{HR}(95 \% \mathrm{Cl})$ & & HR $(95 \% \mathrm{Cl})$ & $\mathrm{HR}(95 \% \mathrm{Cl})$ \\
\hline \multicolumn{10}{|l|}{ Age } \\
\hline Continuous & $63(39)$ & $\begin{array}{l}1.01(0.98- \\
1.05)\end{array}$ & - & $109(98)$ & $\begin{array}{l}0.99(0.97- \\
1.02)\end{array}$ & - & $172(137)$ & $\begin{array}{l}1.01(0.99- \\
1.03)\end{array}$ & - \\
\hline \multicolumn{10}{|l|}{ Gender } \\
\hline \multirow[t]{2}{*}{ Female } & $35(17)$ & 1.00 & 1.00 & $51(45)$ & 1.00 & - & $86(62)$ & 1.00 & - \\
\hline & $34(16)^{*}$ & & & & & & & & \\
\hline \multirow[t]{2}{*}{ Male } & $28(22)$ & $\begin{array}{l}2.01 \\
\quad(1.05-3.84)\end{array}$ & $\begin{array}{l}1.60(0.79- \\
3.24)\end{array}$ & $58(53)$ & $\begin{array}{l}1.15(0.77- \\
1.71)\end{array}$ & & $86(75)$ & $\begin{array}{l}1.40 \\
(1.00-1.97)\end{array}$ & \\
\hline & $27(21)^{*}$ & & & & & & & & \\
\hline \multicolumn{10}{|l|}{ Tumor origin } \\
\hline \multirow[t]{2}{*}{ Intestinal } & & - & - & & - & - & $63(39)$ & 1.00 & 1.00 \\
\hline & & & & & & & $61(37)^{*}$ & & \\
\hline \multirow[t]{2}{*}{$\begin{array}{c}\text { Pancreatobil- } \\
\text { iary type }\end{array}$} & & - & - & & - & - & $109(98)$ & $\begin{array}{l}2.54 \\
\quad(1.73-3.73)\end{array}$ & $1.61(1.01-2.55)$ \\
\hline & & & & & & & $108(98)^{*}$ & & \\
\hline \multicolumn{10}{|l|}{ Tumor size } \\
\hline \multirow[t]{2}{*}{ Continuous } & $63(39)$ & $\begin{array}{l}1.00(0.98- \\
1.02)\end{array}$ & - & $109(98)$ & $\begin{array}{l}1.04 \\
(1.02-1.06)\end{array}$ & $\begin{array}{l}1.02 \\
(1.00-1.04)\end{array}$ & $172(137)$ & $\begin{array}{l}1.02 \\
(1.00-1.03)\end{array}$ & $1.01(0.99-2.55)$ \\
\hline & & & & $108(98)^{*}$ & & & $169(135)^{*}$ & & \\
\hline \multicolumn{10}{|l|}{ T-stage } \\
\hline \multirow[t]{2}{*}{$\mathrm{T} 1-\mathrm{T} 2$} & $16(8)$ & 1.00 & - & $15(10)$ & 1.00 & 1.00 & $31(18)$ & 1.00 & 1.00 \\
\hline & & & & $14(10)^{*}$ & & & $28(16)^{*}$ & & \\
\hline \multirow[t]{2}{*}{ T3-T4 } & $47(31)$ & $\begin{array}{c}1.72(0.79- \\
3.74)\end{array}$ & & $94(88)$ & $\begin{array}{l}2.47 \\
(1.28-4.77)\end{array}$ & $\begin{array}{l}1.06(0.50- \\
2.27)\end{array}$ & 141 (119) & $\begin{array}{l}2.34 \\
\quad(1.42-3.86)\end{array}$ & $1.21(0.66-2.21)$ \\
\hline & & & & $94(88)^{*}$ & & & $141(119)^{*}$ & & \\
\hline \multicolumn{10}{|l|}{ N-stage } \\
\hline \multirow[t]{2}{*}{ NO } & $35(19)$ & 1.00 & - & $32(25)$ & 1.00 & 1.00 & $67(44)$ & 1.00 & 1.00 \\
\hline & & & & $31(25)^{*}$ & & & $64(42)^{*}$ & & \\
\hline \multirow[t]{2}{*}{$\mathrm{N} 1-2$} & $28(20)$ & $\begin{array}{l}1.56(0.83- \\
2.94)\end{array}$ & & $77(73)$ & $\begin{array}{l}2.23 \\
\quad(1.41-3.54)\end{array}$ & $\begin{array}{l}2.06 \\
(1.16-3.65)\end{array}$ & $105(93)$ & $\begin{array}{l}2.19 \\
\quad(1.52-3.16)\end{array}$ & $1.34(0.88-2.05)$ \\
\hline & & & & $77(73)^{*}$ & & & $105(93)^{*}$ & & \\
\hline \multicolumn{10}{|c|}{ Differentiation grade } \\
\hline \multirow{2}{*}{$\begin{array}{l}\text { Well-mod- } \\
\text { erate }\end{array}$} & $31(17)$ & 1.00 & - & $42(32)$ & 1.00 & 1.00 & $73(49)$ & 1.00 & 1.00 \\
\hline & & & & $41(32)^{*}$ & & & $71(48)^{*}$ & & \\
\hline \multirow[t]{2}{*}{ Poor } & $32(22)$ & $\begin{array}{l}1.70(0.90- \\
3.21)\end{array}$ & & $67(66)$ & $\begin{array}{l}2.63 \\
(1.70-4.09)\end{array}$ & $\begin{array}{l}2.05 \\
\quad(1.24-3.39)\end{array}$ & $99(88)$ & $\begin{array}{l}2.24 \\
\quad(1.57-3.18)\end{array}$ & $1.65(1.09-2.48)$ \\
\hline & & & & $67(66)^{*}$ & & & $98(87)^{*}$ & & \\
\hline \multicolumn{10}{|c|}{ Involved margins, status } \\
\hline \multirow[t]{2}{*}{ RO } & $18(5)$ & 1.00 & 1.00 & $7(5)$ & 1.00 & - & $25(10)$ & 1.00 & 1.00 \\
\hline & $17(4)^{*}$ & & & & & & $23(9)^{*}$ & & \\
\hline \multirow[t]{2}{*}{$\mathrm{R} 1$ and $\mathrm{Rx}$} & $45(34)$ & $\begin{array}{l}3.30 \\
\quad(1.29-8.46)\end{array}$ & $\begin{array}{l}2.89(0.94- \\
8.85)\end{array}$ & $102(93)$ & $\begin{array}{l}2.42(0.98- \\
6.00)\end{array}$ & & $147(127)$ & $\begin{array}{l}3.57 \\
\quad(1.87-6.83)\end{array}$ & $2.23(1.10-4.51)$ \\
\hline & $44(33)^{*}$ & & & & & & $146(126)^{*}$ & & \\
\hline Lymphatic grov & & & & & & & & & \\
\hline Absent & $28(10)$ & 1.00 & 1.00 & $35(27)$ & 1.00 & 1.00 & $63(37)$ & 1.00 & 1.00 \\
\hline & $28(10)^{*}$ & & & $34(27)^{*}$ & & & $62(37)^{*}$ & & \\
\hline
\end{tabular}


Table 3 (continued)

\begin{tabular}{|c|c|c|c|c|c|c|c|c|c|}
\hline \multirow{3}{*}{ 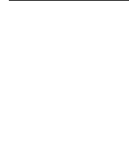 } & \multicolumn{3}{|c|}{ Intestinal type } & \multicolumn{3}{|c|}{ Pancreatobiliary type } & \multicolumn{3}{|l|}{ All } \\
\hline & n (events) & Unadjusted & Adjusted & $\mathrm{n}$ (events) & Unadjusted & Adjusted & n (events) & Unadjusted & Adjusted \\
\hline & & HR (95\% Cl) & HR (95\% Cl) & & HR (95\% Cl) & $\mathrm{HR}(95 \% \mathrm{Cl})$ & & $\mathrm{HR}(95 \% \mathrm{Cl})$ & HR $(95 \% \mathrm{Cl})$ \\
\hline \multirow[t]{2}{*}{ Present } & $35(29)$ & $\begin{array}{l}3.94 \\
(1.90-8.18)\end{array}$ & $\begin{array}{l}1.77(0.77- \\
4.09)\end{array}$ & $74(71)$ & $\begin{array}{l}1.78 \\
(1.13-2.79)\end{array}$ & $\begin{array}{l}1.09(0.65- \\
1.83)\end{array}$ & $109(100)$ & $\begin{array}{l}2.54 \\
\quad(1.73-3.73)\end{array}$ & $1.33(0.85-2.07)$ \\
\hline & $33(27)^{*}$ & & & $74(71)^{*}$ & & & $107(98)^{*}$ & & \\
\hline \multicolumn{10}{|c|}{ Vascular growth } \\
\hline \multirow[t]{2}{*}{ Absent } & $58(34)$ & 1.00 & 1.00 & $73(62)$ & 1.00 & 1.00 & $131(96)$ & 1.00 & 1.00 \\
\hline & $56(32)^{*}$ & & & $72(62)^{*}$ & & & $128(94)^{*}$ & & \\
\hline \multirow[t]{2}{*}{ Present } & $5(5)$ & $\begin{array}{c}6.68(2.40- \\
18.62)\end{array}$ & $\begin{array}{c}4.69(1.48- \\
14.84)\end{array}$ & $36(36)$ & $\begin{array}{l}2.47 \\
\quad(1.61-3.80)\end{array}$ & $\begin{array}{l}2.20 \\
\quad(1.38-3.52)\end{array}$ & $41(41)$ & $\begin{array}{l}3.49 \\
\quad(2.37-5.15)\end{array}$ & $2.18(1.43-3.34)$ \\
\hline & $5(5)^{*}$ & & & $36(36)^{*}$ & & & $41(41)^{*}$ & & \\
\hline \multicolumn{10}{|c|}{ Perineural growth } \\
\hline \multirow[t]{2}{*}{ Absent } & $43(21)$ & 1.00 & 1.00 & $25(19)$ & 1.00 & 1.00 & $68(40)$ & 1.00 & 1.00 \\
\hline & $42(20)^{*}$ & & & $24(19)^{*}$ & & & $66(39)^{*}$ & & \\
\hline \multirow[t]{2}{*}{ Present } & $20(18)$ & $\begin{array}{l}2.81 \\
\quad(1.48-5.35)\end{array}$ & $\begin{array}{l}1.36(0.57- \\
3.21)\end{array}$ & $84(79)$ & $\begin{array}{l}1.96 \\
(1.18-3.25)\end{array}$ & $\begin{array}{l}0.88(0.48- \\
1.61)\end{array}$ & $104(97)$ & $\begin{array}{l}2.96 \\
\quad(2.02-4.43)\end{array}$ & $1.06(0.66-1.71)$ \\
\hline & $19(17)^{*}$ & & & $84(79)^{*}$ & & & $103(96)^{*}$ & & \\
\hline \multicolumn{10}{|c|}{ Growth in peripancreatic fat } \\
\hline \multirow[t]{2}{*}{ Absent } & $42(20)$ & 1.00 & 1.00 & $25(20)$ & 1.00 & 1.00 & $67(40)$ & 1.00 & 1.00 \\
\hline & $40(18)^{*}$ & & & $24(20)^{*}$ & & & $64(38)^{*}$ & & \\
\hline \multirow[t]{2}{*}{ Present } & $21(19)$ & $\begin{array}{l}3.65 \\
(1.86-7.16)\end{array}$ & $\begin{array}{l}1.78(0.73- \\
4.33)\end{array}$ & $84(78)$ & $\begin{array}{l}1.75 \\
\quad(1.06-2.88)\end{array}$ & $\begin{array}{l}0.99(0.56- \\
1.77)\end{array}$ & $105(97)$ & $\begin{array}{l}3.00 \\
(2.03-4.43)\end{array}$ & $1.37(0.85-2.22)$ \\
\hline & $21(19)^{*}$ & & & $84(78)^{*}$ & & & $105(97)^{*}$ & & \\
\hline \multicolumn{10}{|c|}{ Adjuvant treatment } \\
\hline None & $45(30)$ & 1.00 & - & $50(44)$ & 1.00 & - & $95(74)$ & 1.00 & - \\
\hline Any & $18(9)$ & $\begin{array}{l}0.69(0.33- \\
1.47)\end{array}$ & & $59(54)$ & $\begin{array}{l}0.96(0.64- \\
1.42)\end{array}$ & & $77(63)$ & $\begin{array}{l}1.09(0.78-1- \\
53)\end{array}$ & \\
\hline \multicolumn{10}{|l|}{ MMR } \\
\hline \multirow[t]{2}{*}{$\mathrm{pMMR}$} & $49(34)$ & 1.00 & 1.00 & $101(93)$ & 1.00 & 1.00 & $150(127)$ & 1.00 & 1.00 \\
\hline & $49(34)^{*}$ & & & $101(93)^{*}$ & & & $150(127)^{*}$ & & \\
\hline \multirow[t]{2}{*}{$\mathrm{dMMR}$} & $12(3)$ & $\begin{array}{l}0.20 \\
(0.06-0.68)\end{array}$ & $\begin{array}{l}0.36(0.09- \\
1.42)\end{array}$ & $7(5)$ & $\begin{array}{l}0.57(0.23- \\
1.42)\end{array}$ & $\begin{array}{l}1.24(0.46- \\
3.37)\end{array}$ & $19(8)$ & $\begin{array}{l}0.28 \\
(0.13-0.57)\end{array}$ & $0.52(0.23-1.19)$ \\
\hline & $12(3)^{*}$ & & & $7(5)^{*}$ & & & $19(8)^{*}$ & & \\
\hline
\end{tabular}

Italic values indicate significance at $p<0.05$

*Number and events for the adjusted analysis

the nearly equal distribution of treated and untreated patients in this retrospective cohort provides a good setting for analysis of potential treatment predictive markers, despite the retrospective design. A limitation to this cohort is however that performance status (PS), which is an important clinicopathological factor that might have affected weather patients have received adjuvant treatment or not, has not been registered. However, the finding that patients with dMMR tumors who received adjuvant treatment seemed to do worse than those not receiving adjuvant treatment, implies that PS may not have affected the results. Another limitation is related to the use of TMA, which may not reflect the heterogeneity of the tumor, however this is a well-validated method for studies of biomarkers [38]. It should also be pointed out that a single whole tissue section will also merely represent a part of the tumor, and in the herein analyzed TMA, tissue cores were sampled from different archival blocks with primary tumor, and, when present, different lymph node metastases. Therefore, uniform loss of an MMR protein across multiple TMA-samples should provide similarly reliable evidence of $\mathrm{dMMR}$ as analysis of a single whole tissue section. Another issue that needs to be addressed in light of the small sample size is the risk of overfitting the multivariable model. We have however chosen to adjust for known prognostic parameters 


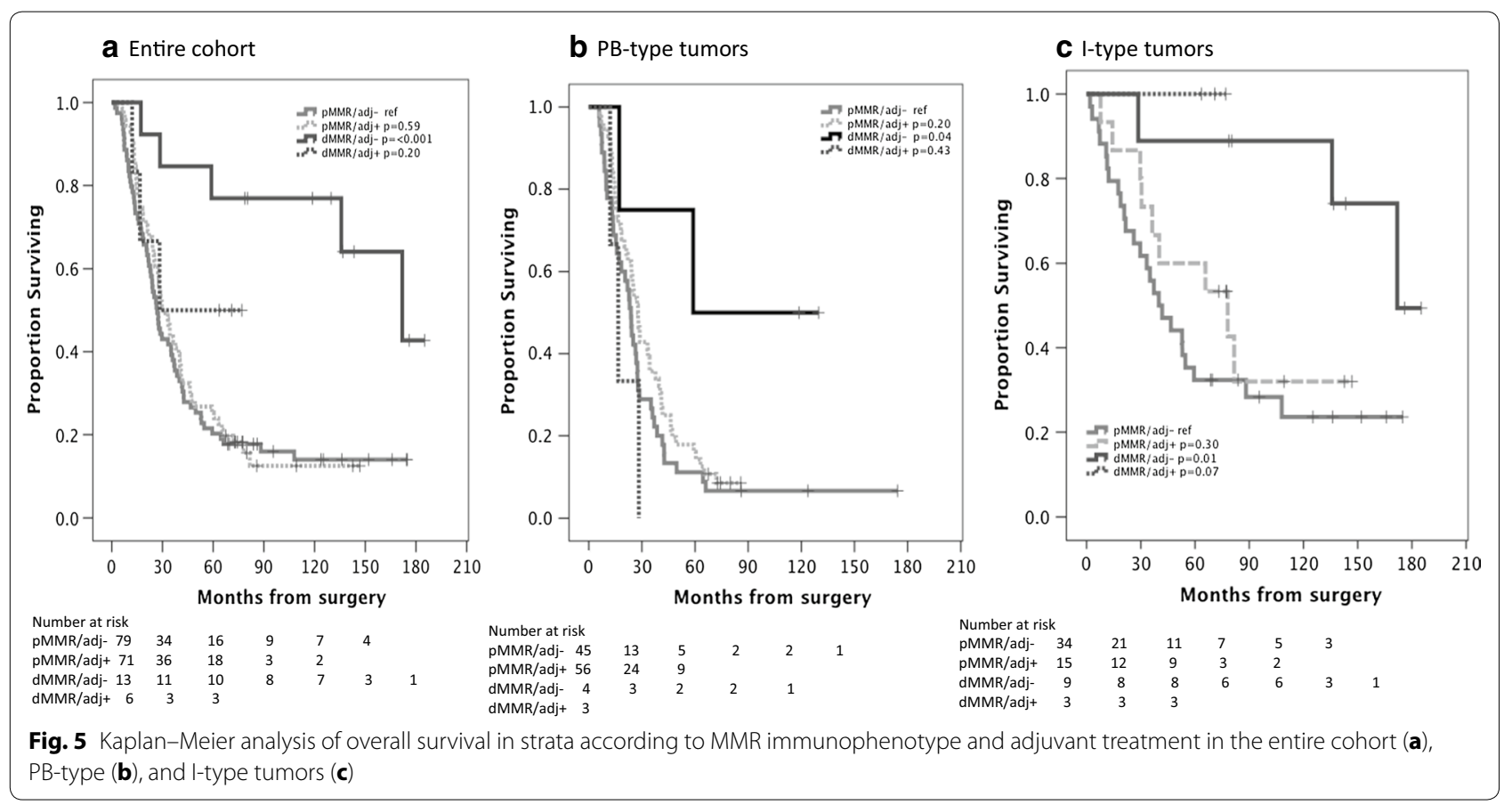

that were also found to be significant in the univariable analysis.

\section{Conclusions}

The results from this study demonstrate that dMMR is more frequent in I-type compared to PB-type periampullary adenocarcinoma, and is a prognostic factor for longterm survival only in the former. However, despite the small numbers, the finding of dMMR being a potential negative predictor of response to adjuvant chemotherapy in PB-type tumors is noteworthy and merits further validation in larger patient cohorts, as it may be highly relevant for clinical decision-making.

\section{Additional file}

Additional file 1. Unadjusted and adjusted hazard ratios of the impact of MMR immunophenotype on recurrence free survival in l-type tumors, PB-type tumors, and the entire cohort.

\section{Abbreviations}

MMR: mismatch repair; dMMR: deficient MMR; PMMR: preserved MMR; PBtype: pancreatobiliary type adenocarcinoma; I-type: intestinal type adenocarcinoma; TMA: tissue microarray; OS: overall survival; RFS: recurrence free survival; HR: hazard ratio; Cl: confidence interval; Q1: quartile 1; Q3: quartile 3.

\section{Authors' contributions}

MH: data acquisition and analysis, writing. SL: data acquisition. BN: methodology. JE: data acquisition. JE: review and editing. KJ: conceptualization, funding acquisition, data acquisition, review and editing. All authors read and approved the final manuscript.
Acknowledgements

Not applicable.

\section{Competing interests}

The authors declare that they have no competing interests.

\section{Availability of data and materials}

All data generated or analyzed during this study are included in this published article.

\section{Consent for publication}

Not applicable.

\section{Ethics approval and consent to participate}

All EU and national regulations and requirements for handling human samples have been fully complied with during the conduct of this project; i.e. Decision No. 1110/94/EC of the European Parliament and of the Council (OJL126 18,5,94), the Helsinki Declaration on ethical principles for medical research involving human subjects, and the EU Council Convention on human rights and Biomedicine. Approval for the study was obtained from the Ethics committee of Lund University (Ref No 445/07), whereby the committee waived no need for consent other than the option to opt out.

\section{Funding}

This study was supported by Grants from the Swedish Research Council (2015-03598, KJ), the Swedish Cancer Society (CAN 2016/483, KJ), the Mrs. Berta Kamprad Foundation (KJ), the Swedish Government Grant for Clinical Research (KJ, JEB), and Lund University Hospital Research Grant (KJ).

\section{Publisher's Note}

Springer Nature remains neutral with regard to jurisdictional claims in published maps and institutional affiliations.

Received: 9 December 2017 Accepted: 5 March 2018

Published online: 14 March 2018 


\section{References}

1. Bronsert $P$, et al. Intestinal-type of differentiation predicts favourable overall survival: confirmatory clinicopathological analysis of 198 periampullary adenocarcinomas of pancreatic, biliary, ampullary and duodenal origin. BMC Cancer. 2013:13:428.

2. Westgaard A, et al. Pancreatobiliary versus intestinal histologic type of differentiation is an independent prognostic factor in resected periampullary adenocarcinoma. BMC Cancer. 2008;8:170

3. Siegel RL, Miller KD, Jemal A. Cancer statistics, 2016. CA Cancer J Clin. 2016;66(1):7-30

4. Yeo CJ, et al. Six hundred fifty consecutive pancreaticoduodenectomies in the 1990s: pathology, complications, and outcomes. Ann Surg. 1997;226(3):248-57 (discussion 257-60)

5. Howe JR, et al. Factors predictive of survival in ampullary carcinoma. Ann Surg. 1998;228(1):87-94.

6. Qiao QL, et al. Carcinoma of the ampulla of Vater: factors influencing long-term survival of 127 patients with resection. World J Surg. 2007:31(1):137-43 (discussion 144-6).

7. Herreros-Villanueva $\mathrm{M}$, et al. Adjuvant and neoadjuvant treatment in pancreatic cancer. World J Gastroenterol. 2012;18(14):1565-72.

8. Gillen S, et al. Preoperative/neoadjuvant therapy in pancreatic cancer: a systematic review and meta-analysis of response and resection percentages. PLoS Med. 2010;7(4):e1000267.

9. Palmer KR, et al. Chemotherapy prolongs survival in inoperable pancreatic carcinoma. Br J Surg. 1994;81(6):882-5.

10. Bakkevold KE, et al. Adjuvant combination chemotherapy (AMF) following radical resection of carcinoma of the pancreas and papilla of Vaterresults of a controlled, prospective, randomised multicentre study. Eur J Cancer. 1993:29A(5):698-703.

11. Royal RE, et al. Phase 2 trial of single agent I pilimumab (anti-CTLA-4) for locally advanced or metastatic pancreatic adenocarcinoma. J Immunother. 2010;33(8):828-33.

12. Johansson $\mathrm{H}_{\text {, et }}$ al. Immune checkpoint therapy for pancreatic cancer. World J Gastroenterol. 2016;22(43):9457-76.

13. Eshleman JR, Markowitz SD. Mismatch repair defects in human carcinogenesis. Hum Mol Genet. 1996;5:1489-94.

14. Chang $L$, et al. Microsatellite instability: a predictive biomarker for cancer immunotherapy. Appl Immunohistochem Mol Morphol. 2017;26:e15-21.

15. Le DT, et al. PD-1 blockade in tumors with mismatch-repair deficiency. $N$ Engl J Med. 2015:372(26):2509-20.

16. Chau I. Clinical development of PD-1/PD-L1 immunotherapy for gastrointestinal cancers: facts and hopes. Clin Cancer Res. 2017;23:6002-11.

17. Fujiyoshi K, et al. Metastatic pattern of stage IV colorectal cancer with high-frequency microsatellite instability as a prognostic factor. Anticancer Res. 2017;37(1):239-47.

18. Riazy M, et al. Mismatch repair status may predict response to adjuvant chemotherapy in resectable pancreatic ductal adenocarcinoma. Mod Pathol. 2015;28(10):1383-9.

19. Eatrides JM, et al. Microsatellite instability in pancreatic cancer. J Clin Oncol. 2016;34(15_suppl):e15753.

20. Nakata B, et al. Prognostic value of microsatellite instability in resectable pancreatic cancer. Clin Cancer Res. 2002;8(8):2536-40.

21. Ruemmele $\mathrm{P}$, et al. Histopathologic features and microsatellite instability of cancers of the papilla of vater and their precursor lesions. Am J Surg Pathol. 2009:33(5):691-704.

22. Elebro J, Jirstrom K. Use of a standardized diagnostic approach improves the prognostic information of histopathologic factors in pancreatic and periampullary adenocarcinoma. Diagn Pathol. 2014;9(1):80
23. Fristedt $R$, et al. Reduced expression of the polymeric immunoglobulin receptor in pancreatic and periampullary adenocarcinoma signifies tumour progression and poor prognosis. PLoS ONE. 2014;9(11):e112728.

24. Elebro J, et al. Expression and prognostic significance of human epidermal growth factor receptors 1, 2 and 3 in periampullary adenocarcinoma. PLOS ONE. 2016;11(4):e0153533.

25. Heby $M$, et al. Prognostic and predictive significance of podocalyxin-like protein expression in pancreatic and periampullary adenocarcinoma. BMC Clin Pathol. 2015:15:10.

26. Elebro J, et al. Prognostic and treatment predictive significance of SATB1 and SATB2 expression in pancreatic and periampullary adenocarcinoma. J Transl Med. 2014;12:289.

27. Lundgren $\mathrm{S}$, et al. The clinical importance of tumour-infiltrating macrophages and dendritic cells in periampullary adenocarcinoma differs by morphological subtype. J Transl Med. 2017;15(1):152.

28. Lundgren $\mathrm{S}$, et al. The prognostic impact of NK/NKT cell density in periampullary adenocarcinoma differs by morphological type and adjuvant treatment. PLoS ONE. 2016:11(6):e0156497.

29. Rahib L, et al. Projecting cancer incidence and deaths to 2030: the unexpected burden of thyroid, liver, and pancreas cancers in the United States. Cancer Res. 2014;74(11):2913-21.

30. Siegel RL, Miller KD, Jemal A. Cancer statistics, 2015. CA Cancer J Clin. 2015;65(1):5-29.

31. Boland CR, et al. The biochemical basis of microsatellite instability and abnormal immunohistochemistry and clinical behavior in Lynch syndrome: from bench to bedside. Fam Cancer. 2008;7(1):41-52.

32. Mangold $\mathrm{E}_{\text {, }}$ et al. Tumours from MSH2 mutation carriers show loss of MSH2 expression but many tumours from MLH1 mutation carriers exhibit weak positive MLH1 staining. J Pathol. 2005:207(4):385-95.

33. Shia J, et al. Value of immunohistochemical detection of DNA mismatch repair proteins in predicting germline mutation in hereditary colorectal neoplasms. Am J Surg Pathol. 2005;29(1):96-104.

34. Maby $\mathrm{P}$, et al. Correlation between density of CD8+T-cell infiltrate in microsatellite unstable colorectal cancers and frameshift mutations: a rationale for personalized immunotherapy. Cancer Res. 2015;75(17):3446-55.

35. Ribic CM, et al. Tumor microsatellite-instability status as a predictor of benefit from fluorouracil-based adjuvant chemotherapy for colon cancer. N Engl J Med. 2003;349(3):247-57.

36. Kim CG, et al. Effects of microsatellite instability on recurrence patterns and outcomes in colorectal cancers. Br J Cancer. 2016;115(1):25-33.

37. Etienne-Grimaldi MC, et al. Molecular patterns in deficient mismatch repair colorectal tumours: results from a French prospective multicentric biological and genetic study. Br J Cancer. 2014;110(11):2728-37.

38. Torhorst J, et al. Tissue microarrays for rapid linking of molecular changes to clinical endpoints. Am J Pathol. 2001:159(6):2249-56.

\section{Submit your next manuscript to BioMed Central and we will help you at every step:}

- We accept pre-submission inquiries

- Our selector tool helps you to find the most relevant journal

- We provide round the clock customer support

- Convenient online submission

- Thorough peer review

- Inclusion in PubMed and all major indexing services

- Maximum visibility for your research

Submit your manuscript at www.biomedcentral com/submit
C Biomed Central 